TITLE:

\title{
Experimental evidence of ion acoustic soliton chain formation and validation of nonlinear fluid theory
}

\section{AUTHOR(S):}

Kakad, Amar; Omura, Yoshiharu; Kakad, Bharati

\section{CITATION:}

Kakad, Amar ... [et al]. Experimental evidence of ion acoustic soliton chain formation and validation of nonlinear fluid theory. Physics of Plasmas 2013, 20(6): 062103.

\section{ISSUE DATE:}

2013-06-14

URL:

http://hdl.handle.net/2433/187946

\section{RIGHT:}

(c) 2013 American Institute of Physics. This article may be downloaded for personal use only. Any other use requires prior permission of the author and the American Institute of Physics. 


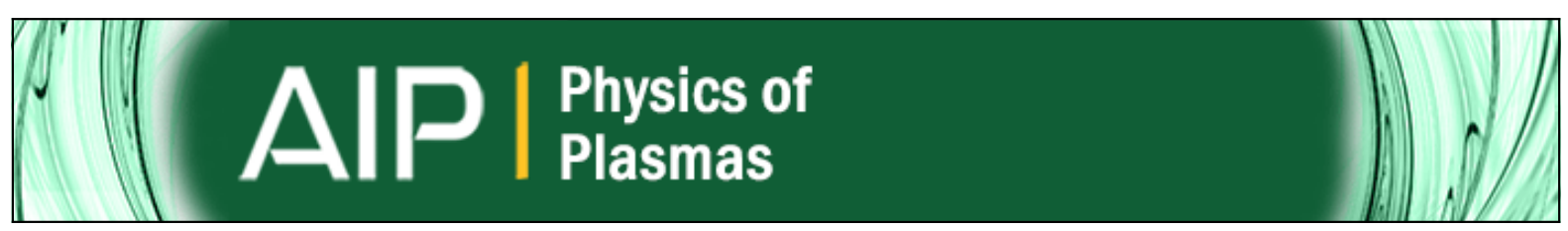

\section{Experimental evidence of ion acoustic soliton chain formation and validation of nonlinear fluid theory}

Amar Kakad, Yoshiharu Omura, and Bharati Kakad

Citation: Physics of Plasmas (1994-present) 20, 062103 (2013); doi: 10.1063/1.4810794

View online: http://dx.doi.org/10.1063/1.4810794

View Table of Contents: http://scitation.aip.org/content/aip/journal/pop/20/6?ver=pdfcov

Published by the AIP Publishing

\section{Articles you may be interested in}

Dust ion acoustic soliton in pair-ion plasmas with non-isothermal electrons

Phys. Plasmas 19, 042304 (2012); 10.1063/1.3696061

Fully kinetic description of the linear excitation and nonlinear saturation of fast-ion-driven geodesic acoustic mode instability

Phys. Plasmas 19, 022102 (2012); 10.1063/1.3680633

Modeling of ion-acoustic soliton excitation through decay process of a localized perturbation

Phys. Plasmas 15, 082105 (2008); 10.1063/1.2965500

Linear and nonlinear ion-acoustic waves in very dense magnetized plasmas

Phys. Plasmas 15, 082303 (2008); 10.1063/1.2965496

Influence of trapped electrons on ion-acoustic solitons in plasmas with superthermal electrons

Phys. Plasmas 14, 012307 (2007); 10.1063/1.2428281

\section{AlP Re-register for Table of Content Alerts}




\title{
Experimental evidence of ion acoustic soliton chain formation and validation of nonlinear fluid theory
}

\author{
Amar Kakad, ${ }^{1,2, a)}$ Yoshiharu Omura, ${ }^{1, b)}$ and Bharati Kakad ${ }^{2, c)}$ \\ ${ }^{1}$ Research Institute for Sustainable Humanosphere, Kyoto University, Uji, Kyoto 611-0011, Japan \\ ${ }^{2}$ Indian Institute of Geomagnetism, New Panvel, Navi Mumbai 410-218, India
}

(Received 18 March 2013; accepted 3 May 2013; published online 14 June 2013)

\begin{abstract}
We perform one-dimensional fluid simulation of ion acoustic (IA) solitons propagating parallel to the magnetic field in electron-ion plasmas by assuming a large system length. To model the initial density perturbations (IDP), we employ a KdV soliton type solution. Our simulation demonstrates that the generation mechanism of IA solitons depends on the wavelength of the IDP. The short wavelength IDP evolve into two oppositely propagating identical IA solitons, whereas the long wavelength IDP develop into two indistinguishable chains of multiple IA solitons through a wave breaking process. The wave breaking occurs close to the time when electrostatic energy exceeds half of the kinetic energy of the electron fluid. The wave breaking amplitude and time of its initiation are found to be dependent on characteristics of the IDP. The strength of the IDP controls the number of IA solitons in the solitary chains. The speed, width, and amplitude of IA solitons estimated during their stable propagation in the simulation are in good agreement with the nonlinear fluid theory. This fluid simulation is the first to confirm the validity of the general nonlinear fluid theory, which is widely used in the study of solitary waves in laboratory and space plasmas. C 2013 AIP Publishing LLC. [http://dx.doi.org/10.1063/1.4810794]
\end{abstract}

\section{INTRODUCTION}

Solitary waves (or solitons) are the localized structures that arise due to the balance between nonlinearity and the dispersive effects in the medium. They are observed in many physical systems, including water, ${ }^{1}$ plasma, ${ }^{2}$ liquid helium, ${ }^{3}$ and optics. ${ }^{4}$ Especially, they have been studied intensively for decades in regard to a broad range of physical problems related to space ${ }^{5-7}$ and laboratory plasmas. ${ }^{8-10}$

In plasmas, the solitary waves are observed as isolated nonlinear phenomena exhibiting either unipolar, bipolar, or tripolar pulses in the electric field. Among these, the bipolar electric field pulses are generally observed in plasmas and are recognized as electron or ion solitary waves. Theoretically, they are interpreted as either Bernstein-Greene-Kruskal (BGK) electron/ion phase space holes ${ }^{11-13}$ or electron/ion acoustic (IA) solitons. ${ }^{14-17}$

BGK phase space holes consist of vortices in electron/ ion velocity space due to the trapping of particles in an electric potential and are described by solitary-wave solutions of nonlinear Vlasov-Poisson equations in a collisionless plasma. ${ }^{18}$ Based on the particle simulation, ${ }^{5,19}$ it was proposed that these solitary waves can be generated by the nonlinear BGK mode attained from an electron beam instability. Other generation mechanisms for the solitary waves are based on the nonlinear evolution of electron two stream instabilities. $^{20-22}$

The electron and ion acoustic soliton models are based on the multi-fluid approach. There are numerous contributions to the theory of multiple-species plasma for the ion and

\footnotetext{
a)amar@iigs.iigm.res.in

b) omura@rish.kyoto-u.ac.jp

c)ebharati@iigs.iigm.res.in
}

electron acoustic waves within the context of the fluidPoisson equations. The critical Mach numbers predicted by these models are based on isothermal and adiabatic treatments of the electrons. ${ }^{23,24}$ Most of the soliton models are based on the reductive perturbation technique, which makes it possible to reduce governing nonlinear equations to the Korteweg-de Vries (KdV) equation. ${ }^{25-27}$ The solutions of the $\mathrm{KdV}$ equation represent small amplitude electron or ion acoustic solitons. This technique is based on the assumption that the amplitude of the wave is small, and thus cannot be applied to the study of large amplitude solitary waves. Furthermore, a number of attempts have been made in the past studies to solve the initial-value problem of the $\mathrm{KdV}$ equation for the prediction of exact solitons. ${ }^{28,29}$

Other multi-species plasma models are based on the pseudo potential approach, which is renowned and has been extensively used in the study of stationary arbitrary amplitude solitary waves. ${ }^{16,24,30-40}$ Although these models predict the Mach number (soliton speed) range for the existence of stationary solitons, they do not provide information on their generation mechanisms and time evolutionary dynamics.

To address these issues, we carry out one-dimensional fluid simulations to investigate generation and evolution of nonlinear IA solitary waves in an electron-ion warm plasma. This paper is structured as follows. The model equations and the numerical scheme used for development of the simulation code are given in Sec. II. The simulation results are discussed in Sec. III. The nonlinear fluid theory for IA solitary waves is described in Sec. IV. In Sec. V, the results obtained from the fluid simulations are compared with the nonlinear fluid theory. A phenomenon of wave breaking is discussed in Sec. VI and the results are concluded in Sec. VII. 


\section{BASIC SET OF EQUATIONS AND COMPUTATION METHODS}

We consider a homogeneous, collisionless two-component plasma consisting of fluid electrons and fluid ions $\left(\mathrm{H}^{+}\right.$ions $)$. For the nonlinear IA waves propagating parallel to the magnetic field, the dynamics of the electrons and ions is governed by the multi-fluid equations of continuity, momentum, and energy of each species, and the Poisson equation as follows:

$$
\begin{gathered}
\frac{\partial n_{j}}{\partial t}+\frac{\partial\left(n_{j} v_{j}\right)}{\partial x}=0 \\
\frac{\partial v_{j}}{\partial t}+v_{j} \frac{\partial v_{j}}{\partial x}+\frac{1}{\mu_{j} n_{j}} \frac{\partial P_{j}}{\partial x}-\frac{Z_{j}}{\mu_{j}} E=0 \\
\frac{\partial P_{j}}{\partial t}+v_{j} \frac{\partial P_{j}}{\partial x}+\gamma_{j} P_{j} \frac{\partial v_{j}}{\partial x}=0 \\
\frac{\partial E}{\partial x}=n_{i}-n_{e}
\end{gathered}
$$

The electric field $(E)$ in the above set of equations can be written in terms of an electrostatic potential $(\phi)$ with the relation, $E=-\partial \phi / \partial x$. In the equations listed above, the subscripts $j=e$ and $i$ are, respectively, used for electrons and ions. The variables $n_{j}, P_{j}$, and $v_{j}$ are plasma density, pressure, and velocity of the species $j$, respectively. Here, $\mu_{j}=m_{j} / m_{i}$, where $m_{j}$ and $Z_{j}$, respectively, represent the mass and charge of the $j$ th species (i.e., $Z_{e}=-$ e for electron and $Z_{i}=e$ for ions). Further, densities are normalized with the background ion density $n_{i 0}$, velocities with the ion thermal velocity $C_{i}=\left(T_{i} / m_{i}\right)^{1 / 2}$, time with the inverse of ion plasma frequency $\omega_{p i}^{-1}$, length with the ion Debye length, electric fields $E$ with $T_{i} / e \lambda_{d i}$, and thermal pressures $P_{j}$ with $n_{0} T_{i}$. Here, $n_{i 0}=n_{e 0}=n_{0}, \omega_{p i}=\sqrt{n_{0} e^{2} / \epsilon_{0} m_{i}}$, and $\lambda_{d i}=\sqrt{\epsilon_{0} T_{i} / n_{0} e^{2}}$. We assume the same adiabatic index, i.e., $\gamma_{j}=3$, for all the species in the equation of state given by Eq. (3). Since we deal with a one-dimensional problem, the particles have one degree of freedom along the wave propagation direction and hence we choose $\gamma=3$ for both species. ${ }^{24,34}$

In the simulation, the spatial derivatives of the quantities in Eqs. (1)-(4) are computed using the finite difference scheme, which is accurate to the fourth order ${ }^{41,42}$

$$
\frac{\partial F_{h}}{\partial x}=\frac{8\left(F_{h+1}-F_{h-1}\right)-F_{h+2}+F_{h-2}}{12 \Delta x},
$$

where $\Delta x$ is grid spacing; and $F_{h}$ represent a physical quantity defined at grid " $h$ ". We integrate the Eqs. (1)-(3) in time with time step $\Delta t$ by the leap-frog method, ${ }^{42}$ which is accurate to the second order. The leap-frog method gives rise to a grid separation numerical instability. To eliminate small wavelength modes linked with such numerical instability, a compensated filter ${ }^{42}$ with the following form is used in the simulation:

$$
F_{h}^{*}=\frac{5}{8} F_{h}+\frac{1}{4}\left(F_{h-1}+F_{h+1}\right)-\frac{1}{16}\left(F_{h-2}+F_{h+2}\right),
$$

where $F_{h}^{*}$ is a filtered physical quantity $F$ at grid " $h$ ".

We perform the fluid simulations in a one-dimensional system with the periodic boundary conditions. For all simulation runs, we assume an artificial electron-to-ion mass ratio $\mu_{e}=0.01$ and electron-to-ion temperature ratio $T_{r}=T_{e} / T_{i}=5$. The flow velocities of electrons and ions at $t=0$ are assumed to be zero, i.e., $v_{e}(x)=v_{i}(x)=0$. The background electron and ion densities are set to one, i.e., $n_{e 0}=n_{i 0}=1$. These background densities are superimposed by a localized perturbation of the following form:

$$
\delta n=\Delta n \operatorname{sech}^{2}\left(\frac{x-x_{c}}{l_{0}}\right) .
$$

In the above equation, $\Delta n$ and $l_{0}$ give the amplitude and width of the superimposed density perturbation. Here, $x_{c}$ is the center of the simulation system. Thus, the perturbed densities $n_{j}(x)=n_{j 0}+\delta n$ take the following form at $t=0$

$$
n_{j}(x)=n_{j 0}+\Delta n \operatorname{sech}^{2}\left(\frac{x-x_{c}}{l_{0}}\right) .
$$

The initial density perturbation (IDP) $\delta n$ considered here has a similar form as the analytical solution of the $\mathrm{KdV}$ equation. $^{27}$ We also compute the strength of IDP using the following equation:

$$
S=\int_{0}^{L_{x}} \delta n d x .
$$

Here, $L_{x}$ is the length of the simulation system. We divide the IDP in two categories based on whether the wavelengths linked with them are larger or smaller as compared to the electron Debye length. We have performed simulation runs for (a) short wavelength $\left(k^{2} \lambda_{d e}^{2}>1\right)$ and (b) long wavelength $\left(k^{2} \lambda_{d e}^{2}<1\right)$ IDPs. Here, $k$ and $\lambda_{d e}$ are the wave number and the electron Debye length, respectively. For short and long wavelength IDPs, we use $l_{0}=10, \Delta n=0.2,0.5,1$ and $l_{0}=60,100, \Delta n=0.2,1$, respectively. Other parameters of the simulation runs under these categories are provided in Table I. Examples of short $\left(l_{0}=10, \Delta n=0.5\right)$ and long $\left(l_{0}=100, \Delta n=1\right)$ wavelength IDPs are shown in Figure 1 along with their Fourier spectra in $k$-space. The vertical dashed line shown in Figure 1(a) represents $k^{2} \lambda_{d e}^{2}=1$ (gives $k=0.45 \lambda_{d i}^{-1}$ ), which categorizes the IDPs in short and long wavelength domains for $T_{r}=5$. It is noted that the short(long) wavelength IDP takes larger(smaller) $k$-space.

\section{SIMULATION RESULTS}

\section{A. Short wavelength density perturbation $\left(k^{2} \lambda_{d e}^{2}>1\right)$}

Here, we discuss the generation and evolution of IA solitary structures when the short wavelength type IDP is TABLE I. Simulation parameters for different run.

\begin{tabular}{lccccc}
\hline \hline Run & $\begin{array}{c}\Delta x \\
{\left[\lambda_{d i}\right]}\end{array}$ & $\begin{array}{c}\Delta t \\
{\left[\omega_{p i}^{-1}\right]}\end{array}$ & $\begin{array}{c}L_{x} \\
{\left[\lambda_{d i}\right]}\end{array}$ & $\begin{array}{c}l_{0} \\
{\left[\lambda_{d i}\right]}\end{array}$ & $\begin{array}{c}\Delta n \\
{\left[n_{0}\right]}\end{array}$ \\
\hline 1 & 0.4 & 0.001 & $3 \mathrm{e} 10^{5}$ & 10 & 0.2 \\
2 & 0.4 & 0.001 & $3 \mathrm{e} 10^{5}$ & 10 & 0.5 \\
3 & 0.4 & 0.001 & $3 \mathrm{e} 10^{5}$ & 10 & 1.0 \\
4 & 0.4 & 0.001 & $3 \mathrm{e} 10^{5}$ & 60 & 0.2 \\
5 & 0.4 & 0.001 & $4 \mathrm{e} 10^{5}$ & 100 & 0.2 \\
6 & 0.4 & 0.001 & $3 \mathrm{e} 10^{5}$ & 60 & 1.0 \\
7 & 0.4 & 0.001 & $4 \mathrm{e} 10^{5}$ & 100 & 1.0 \\
\hline \hline
\end{tabular}




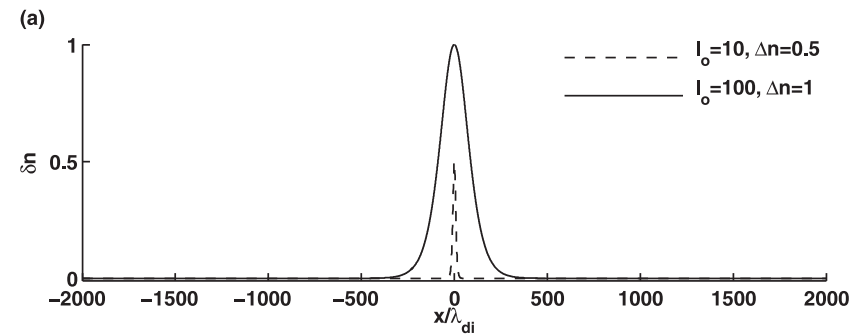

(b)

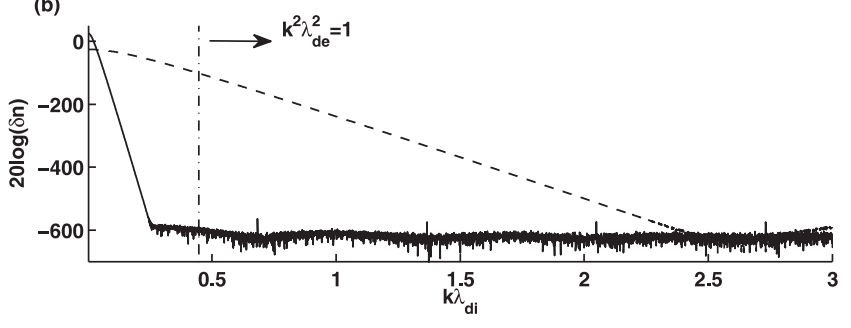

FIG. 1. (a) Graphical representation of IDP $\delta n$ for short wavelength $\left(k^{2} \lambda_{d e}^{2}>1\right)$ case $l_{0}=10, \Delta n=0.5$ and long wavelength $\left(k^{2} \lambda_{d e}^{2}<1\right)$ case $l_{0}=100, \Delta n=1$. (b) Fourier spectrum of these density perturbations in $k$-space. The vertical dashed line corresponds to the limit $k^{2} \lambda_{d e}^{2}=1$, which gives $k=0.45 \lambda_{d i}^{-1}$ for $T_{r}=5$ and divides the perturbations in short and long wavelength regimes.

introduced to the background electron and ion densities. Under this category, we perform three simulation runs for perturbation strengths $S=4$ (Run-1), 10 (Run-2), and 20 (Run-3). Some of the steps during formation and evolution of IA pulses are demonstrated in Figure 2. Perturbed densities $n_{j}(\mathrm{x})$ at $\omega_{p i} t=0$ are shown in Figure 2(a). This density perturbation evolves into two unstable IA pulses and two Langmuir wave packets at $\omega_{p i} t=t_{1}$, which is depicted in Figure 2(b). In this case, Langmuir wave packets propagate with a group speed $\left(V_{g}\right)$ greater than that of IA solitary pulses and moves towards the boundaries of the simulation system. The unstable IA pulses follow the Langmuir wave packets and travel with a speed $V_{s}$. During the course of evolution, IA oscillations are developed at the inner edge of these unstable IA pulses as shown in Figure 2(c). Thus Langmuir waves, IA pulses, and IA oscillations propagate with speeds $V_{g}, V_{s}$, and $V_{s 0}$, respectively, such that $V_{s 0}<V_{s}<V_{g}$. Langmuir waves dissipate considerably with time by losing their energy to the IA oscillations. At time $\omega_{p i} t=t_{3}$, Langmuir wave packets, IA solitary pulses, and IA oscillations are distinct and reasonably isolated from each other, which is shown in Figure 2(d). The IA solitary pulses do not change their amplitudes, widths, and speeds after time $\omega_{p i} t=t_{3}$, hence they are termed as IA solitons.

Under this category, we discuss the evolution of IA pulses for $l_{0}=10, \Delta n=0.5$, and $S=10$ (Run-2). We examine the evolution and propagation of different wave structures through spatial and temporal evolution of their electrostatic potentials, which is depicted in Figure 3. It should be noted that we use a large system length $\left(L_{x}=300000 \lambda_{d i}\right)$ for the present simulation run and hence only the part of the system from $x=-3500 \lambda_{d i}$ to $3500 \lambda_{d i}$ is shown in this Figure 3 . Here, $x=0$ represents the center of the simulation system. The horizontal dotted line shown in Figure 3 at $\omega_{p i} t=10$ corresponds to the time of formation of two unstable IA pulses. After their formation, these IA pulses propagate opposite to each other and are seen as two red bands. The blue bands represent the propagation of IA oscillations. The inset shown in Figure 3 clearly shows the propagation of Langmuir wave with distinct wave structures. The speed obtained from their propagation path indicates that these packets propagate in a direction opposite to each other with group velocity less than $\sqrt{3} C_{e}$, where $C_{e}=\sqrt{T_{e} / m_{e}}$ is the

\section{Short wavelength density perturbation $\left(k^{2} \lambda_{d e}^{2}>1\right)$}

\section{(a) Initial density perturbation, $\omega_{p i} t=0$}

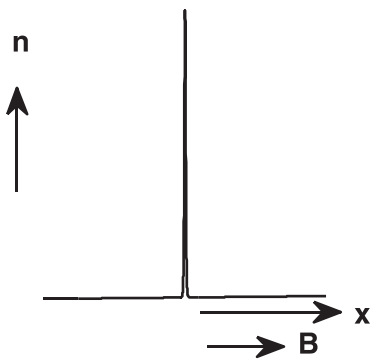

(c) Evolution of solitary pulses, $\omega_{p i} t^{t} t_{2}$

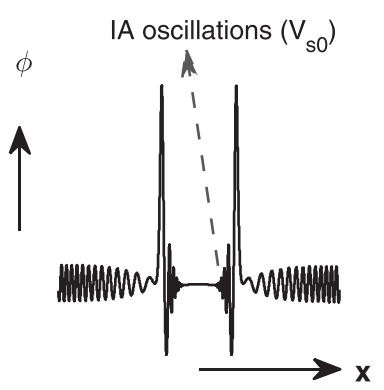

(b) Formation of two IA solitary pulses, $\omega_{p i} t=t_{1}$

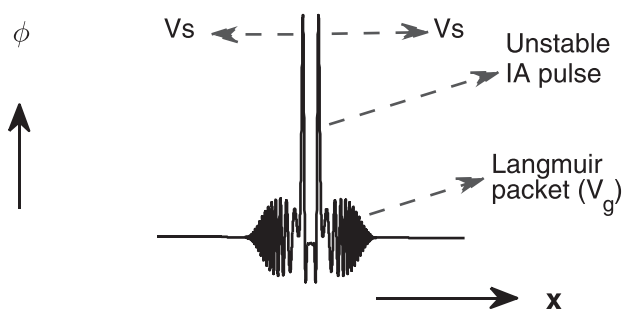

(d) Formation of stable solitons, $\omega_{p i} t=t_{3}$

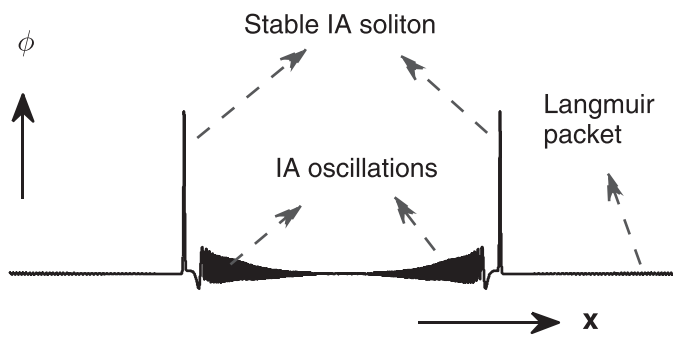

FIG. 2. Schematic diagrams showing some of the steps during formation and evolution of IA pulses for the case of short wavelength IDP. 


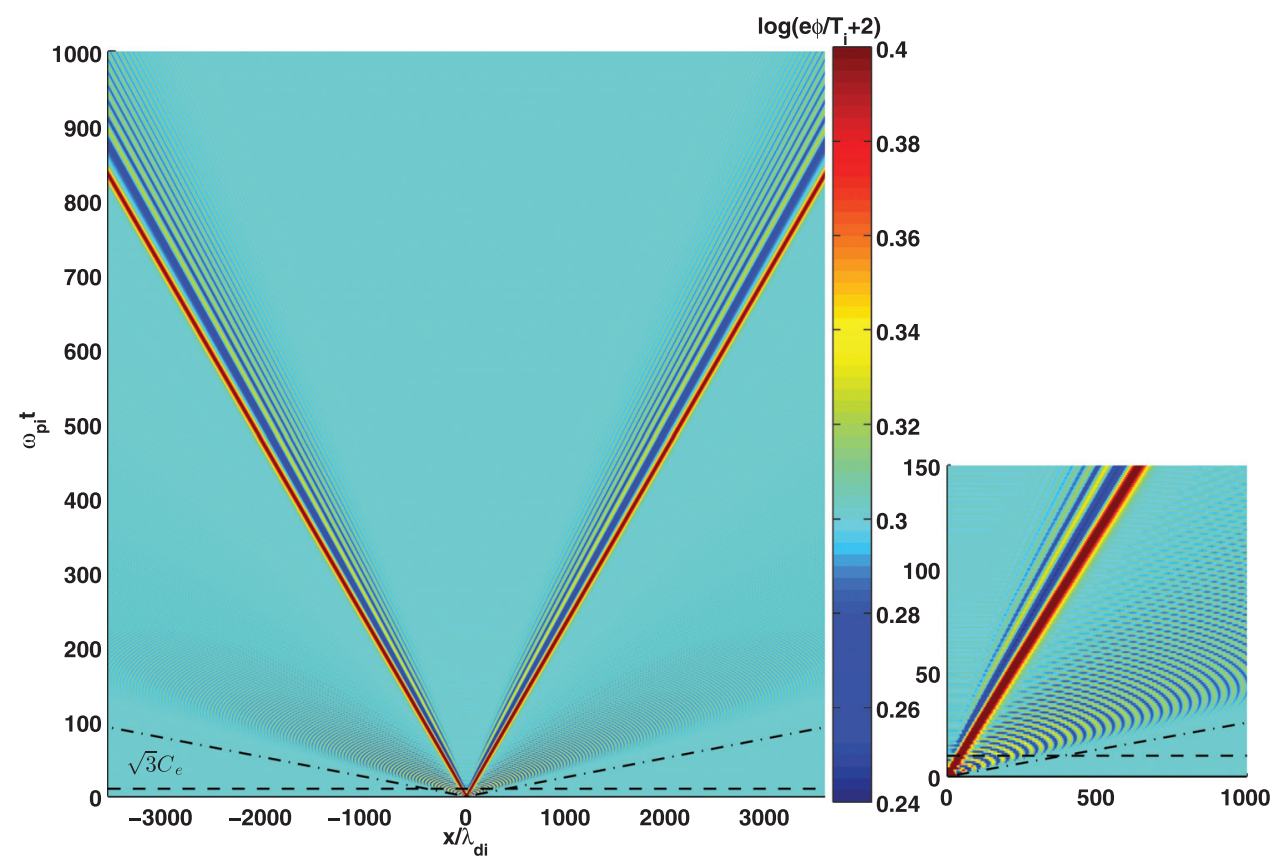

FIG. 3. Spatial and temporal evolution of electrostatic potentials for $l_{0}=10$, $\Delta n=0.5$ (Run-2). The upper dark blue bands represent the propagation path of IA oscillations. The dark red bands correspond to IA pulses propagating opposite to each other. The inset shows Langmuir wave packet with distinct wave structures.

electron thermal speed. The presence of the IA and Langmuir modes can be confirmed from dispersion diagrams. The $\omega-k$ diagrams obtained by Fourier transforming the electric field in space and time for periods (a) $\omega_{p i} t=0-20$ and (b) $\omega_{p i} t=80-100$ are shown in Figure 4. It is noted that the energy linked with IDP flows into Langmuir and IA modes developed in the system as diagnosed in Figure 4. The numerical velocity $(\Delta x / \Delta t)$ is higher than the phase velocities of these normal modes in the system. The lower dispersion curves are for IA modes, whereas the dispersion curves starting at $\omega / \omega_{p i} \geq 10$ correspond to the Langmuir modes and their harmonics. The speed of the IA mode obtained from its spatial and temporal variations and the speed of the Langmuir mode obtained from its linear dispersion relation (i.e., $\sqrt{3} T_{e} / m_{e}$ ) are shown by dotted lines in Figure 4. It should be noted that the Langmuir harmonics weaken at later time.

After time $\omega_{p i} t=4400$, the Langmuir waves, IA pulses, and the IA oscillations are well separated from each other, and the properties of IA pulses are found to be unchanged. In Figure 5, we show the snapshots of electron and ion perturbed densities $\left(n_{e}, n_{i}\right)$, electrostatic potential $(\phi)$, and electric field $(E)$ associated with the IA pulses at time $\omega_{p i} t=4400$ and $\omega_{p i} t=5400$ for Run-2. The densities, electric field, and potential shown in Figure 5 are associated with the Langmuir wave, IA soliton, and IA oscillations that are propagating in positive $x$-direction. It is seen that the electric field and the electrostatic potential associated
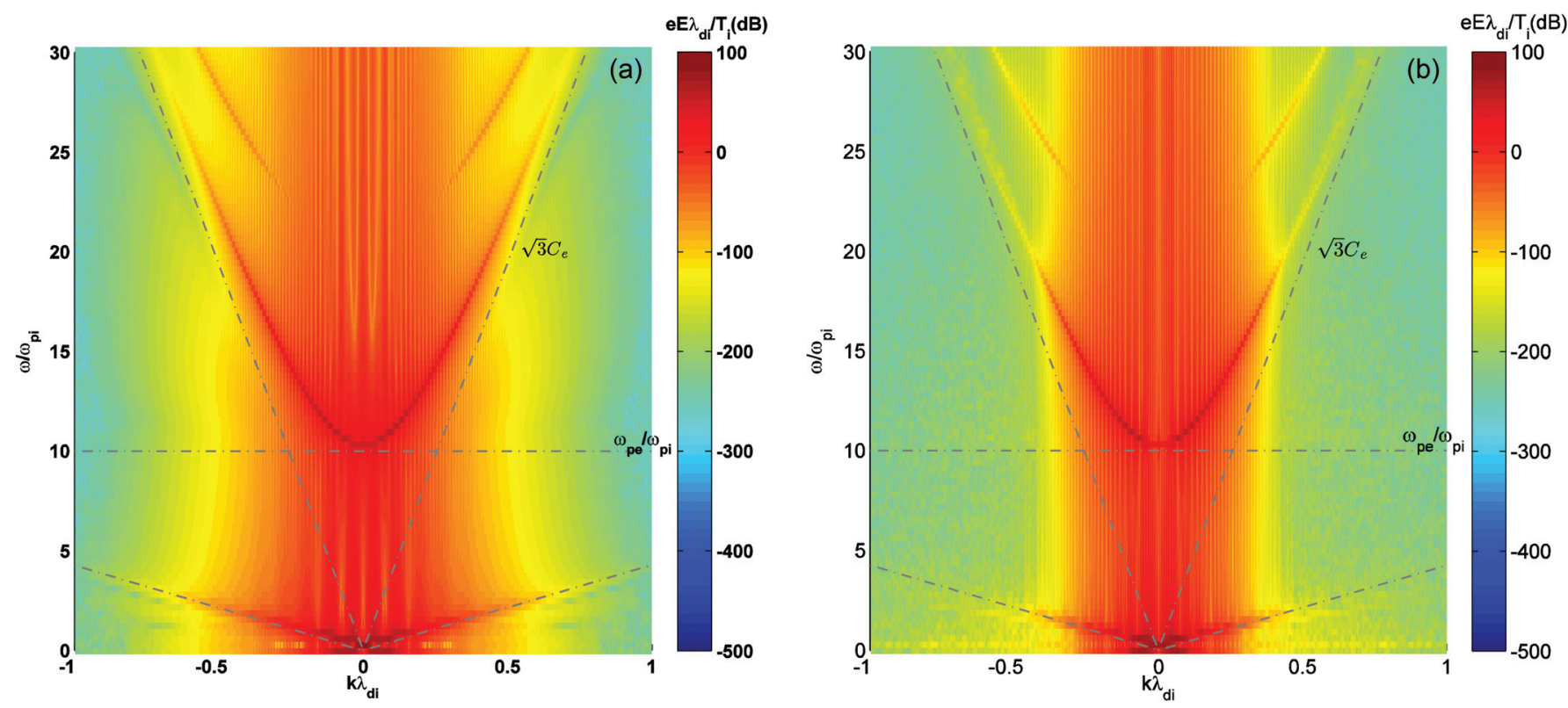

FIG. 4. $\omega-k$ dispersion diagrams during (a) $\omega_{p i} t=0-20$ and (b) $\omega_{p i} t=80-100$ for $l_{0}=10, \Delta n=0.5$ (Run-2). It shows presence of both Langmuir and IA modes. The speed of Langmuir mode (IA mode) estimated from their standard linear dispersion equations (spatial temporal propagation) are shown with upper (lower) slanted dashed lines. The electron plasma frequency is shown with horizontal black dotted line at $\omega_{p e} / \omega_{p i}=10$. 


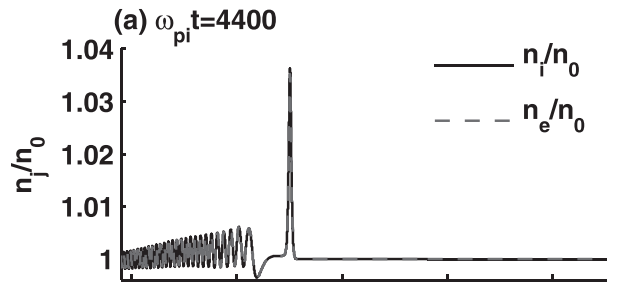

(b) $\omega_{p i} t=4400$

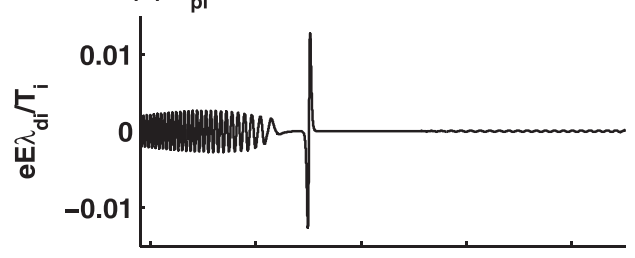

(c) $\omega_{p i} t=4400$

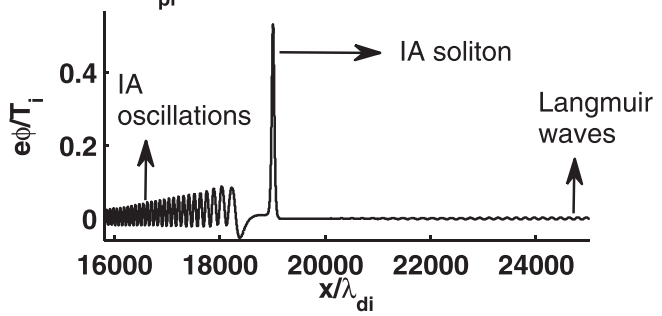

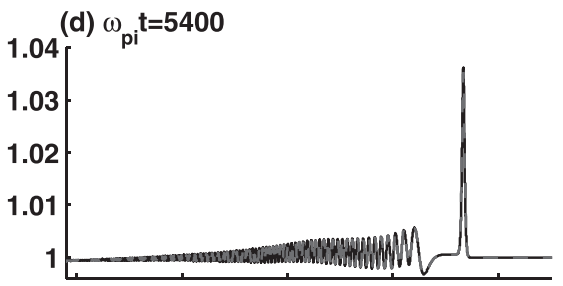

(e) $\omega_{\mathrm{pi}} \mathrm{t}=5400$

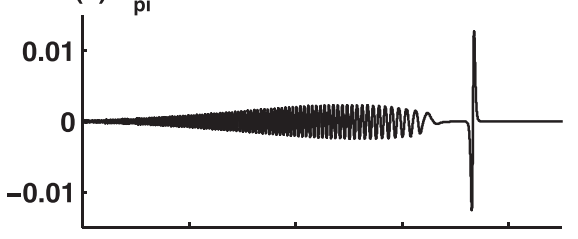

(f) $\omega_{p i} t=5400$



FIG. 5. Snapshots of (a) electron and ion densities (b) electric field and (c) electrostatic potential associated with IA soliton for $\omega_{p i} t=4400$ for Run-2. The same parameters are shown in (d)-(f) for $\omega_{p i} t=5400$. Here, the profiles are associated with the solitary pulse propagating in positive $x$-direction. with IA pulses are nearly the same during their propagation from $\omega_{p i} t=4400-5400$, which confirms the stability of IA pulses, and thus we term them as IA solitons. In the stability region, the average amplitude $\left\langle\phi_{m}\right\rangle$, width $\langle w\rangle$, and speed $\left\langle V_{s}\right\rangle$ of IA soliton and IA oscillation speed $\left\langle V_{s 0}\right\rangle$ are found to be $0.53,56.4,4.32$, and 4.20 , respectively. The simulation data for Run-1 and Run- 3 are also analyzed, and the average estimates of their properties are given in Table II.

\section{B. Long wavelength density perturbation $\left(k^{2} \lambda_{d e}^{2}<1\right)$}

Here, we discuss the generation and evolution of IA pulses when the long wavelength type IDP is used to perturb

TABLE II. IA soliton properties during their stability from fluid simulations along with corresponding estimates from nonlinear fluid theory.

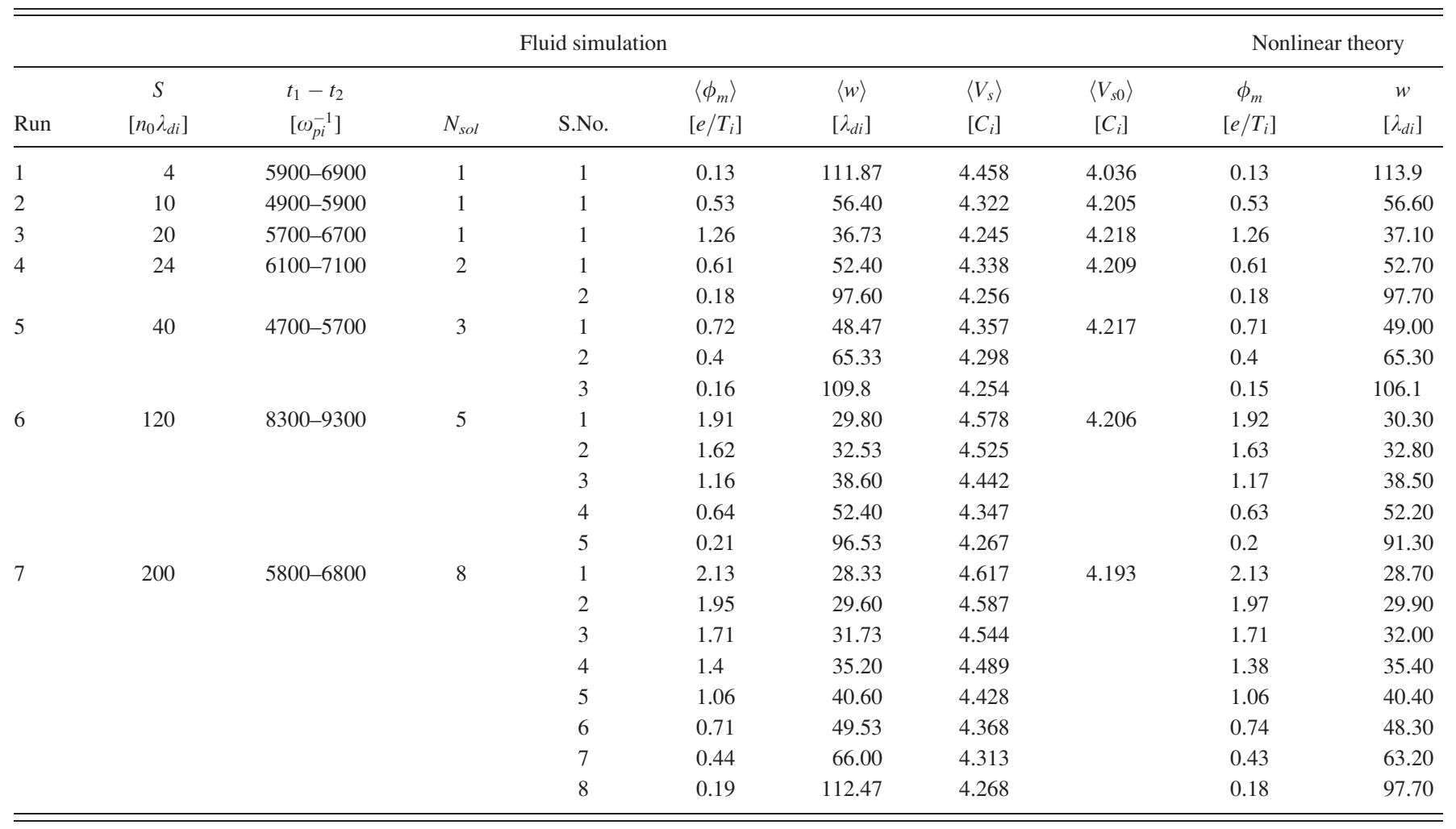


the background electron and ion densities. It is seen that for the long wavelength IDP, two chains of multiple solitons are formed through a process of wave breaking. Some of the time evolutionary characteristics of IA solitons are discussed by schematic diagrams in Figure 6. The perturbed densities of electron and ion $n_{j}(x)$ at $t=0$ for the long wavelength IDP is shown in Figure 6(a). This density perturbation evolves into two unstable IA solitary pulses and the Langmuir wave packets as shown in Figure 6(b). These solitary pulses are indistinguishable and propagate towards the opposite boundaries of the simulation system with the same speed. Both Langmuir wave packets propagate with group velocities greater than the speed of IA solitary pulses. During the course of propagation of these unstable IA pulses, it is seen that their amplitude and speed increases and the trailing edges tend to steepen. As a result of this steepening process, the IA solitary pulses acquire a critical amplitude, which is shown in Figure 6(c). The amplitudes of IA pulse cannot increase further and both solitary pulses collapse through the wave breaking process that is shown in Figure 6(d). Once initiated, this wave breaking process continues and two chains of solitary pulses are generated in the simulation system. A larger amplitude solitary pulse in the chains travels with a greater speed while a smaller amplitude solitary pulse propagates with a less speed. At later stage, these IA solitary structures form distinct chain of stable solitons, which maintain their properties for a long time. These stable chains of IA solitons are shown in Figure 6(e) along with IA oscillations, which are developed during the course of evolution and follow the smallest amplitude soliton in the chain. Under this category, we perform four simulation runs for perturbation strengths $S=24$ (Run-4), 40 (Run-5), 120
(Run-6), and 200 (Run-7). Here, we discuss the Run-7 in detail. The parameters considered for this run are $L_{x}=400000 \lambda_{d i}, l_{0}=100$, and $\Delta n=1$. Spatial and temporal evolution of electrostatic potential associated with different modes is illustrated in Figure 7. The horizontal dotted line at $\omega_{p i} t=90$ indicates the time of formation of two unstable IA pulses, whereas the horizontal dotted line at $\omega_{p i} t=210$ corresponds to the initiation of the wave breaking. It is seen that number of red bands in Figure 7 start increasing after the initiation of wave breaking. This shows that the initially formed unstable IA pulses break into multiple IA pulses, which are observed as two chains of stable multiple IA solitons at later time. The development of IA oscillations is also seen (blue bands in Figure 7) at the inner edge of this solitary chain. Each solitary chain formed in this run contains eight number of solitons $\left(N_{s o l}=8\right)$. Figure 7 shows the spatio-temporal evolution till $\omega_{p i} t=800$. The formation of unstable pulses and their evolution into stable IA solitons is a long time process; thus, we see only five IA solitary pulses in Figure 7. The propagation of the Langmuir waves with distinct wave structures is clearly visible in the inset of Figure 7 . The slanted dotted lines shown in Figure 7 represents the speed $\sqrt{3} C_{e}$. Thus, it is found that the Langmuir wave packets travels with a group velocity less than $\sqrt{3} C_{e}$, which is in agreement with the linear dispersion of the Langmuir wave. ${ }^{43}$

For Run-7, the dispersion diagrams obtained by Fourier transforming the electric field in space and time for time $\omega_{p i} t=20-40, \omega_{p i} t=80-100, \omega_{p i} t=160-180$, and $\omega_{p i} t=$ $240-260$ are, respectively, shown in Figures 8(a)-8(d). Figure 8 (a) show the $\omega-k$ diagram during early phase of evolution, whereas Figure 8(b) display $\omega-k$ plot after formation of two unstable IA pulses. Similarly, Figures 8(c) and 8(d) illustrate

Long wavelength density perturbation $\left(\mathrm{k}^{2} \lambda_{\mathrm{de}}^{2}<1\right)$

(a) Initial density perturbation, $\omega_{p i} t=0$

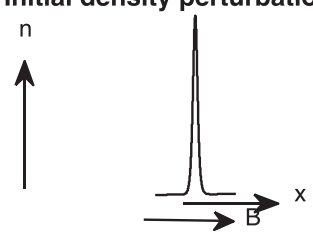

(c) Steepening of solitary pulses, $\omega_{p i} t=t_{2}$

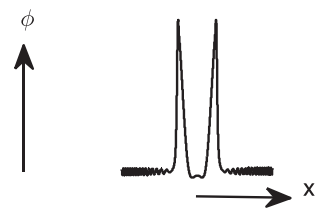

(b) Formation of two solitary pulses, $\omega_{\mathrm{pi}} \mathrm{t}=\mathrm{t}_{1}$

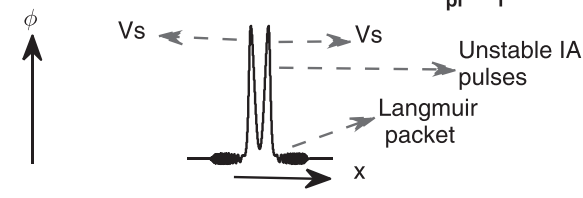

(d) Wave breaking of solitary pulses, $\omega_{\mathrm{pi}} \mathrm{t}=\mathrm{t}_{3}$

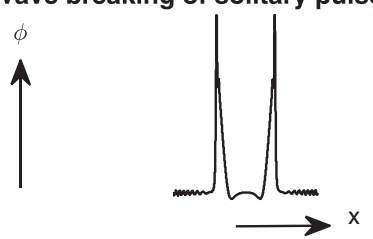

FIG. 6. Schematic diagrams illustrate some of the steps during formation and evolution of chains of multiple IA solitons in case of long wavelength IDP.

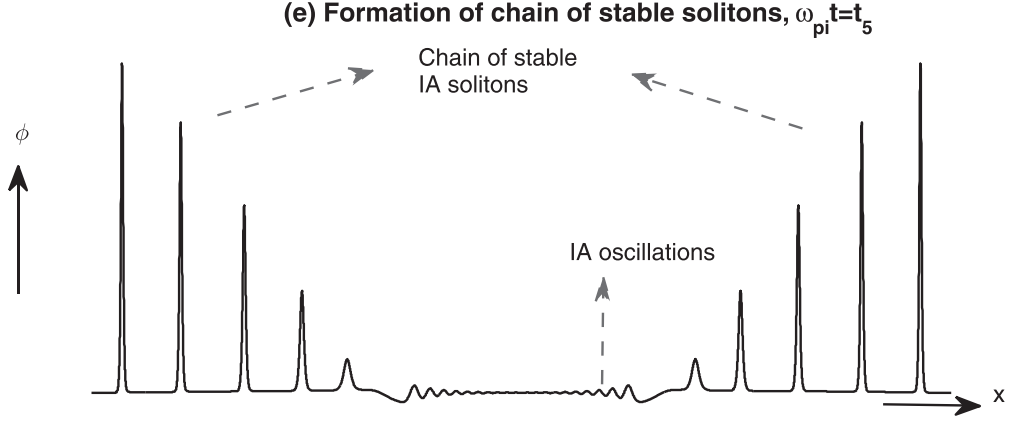



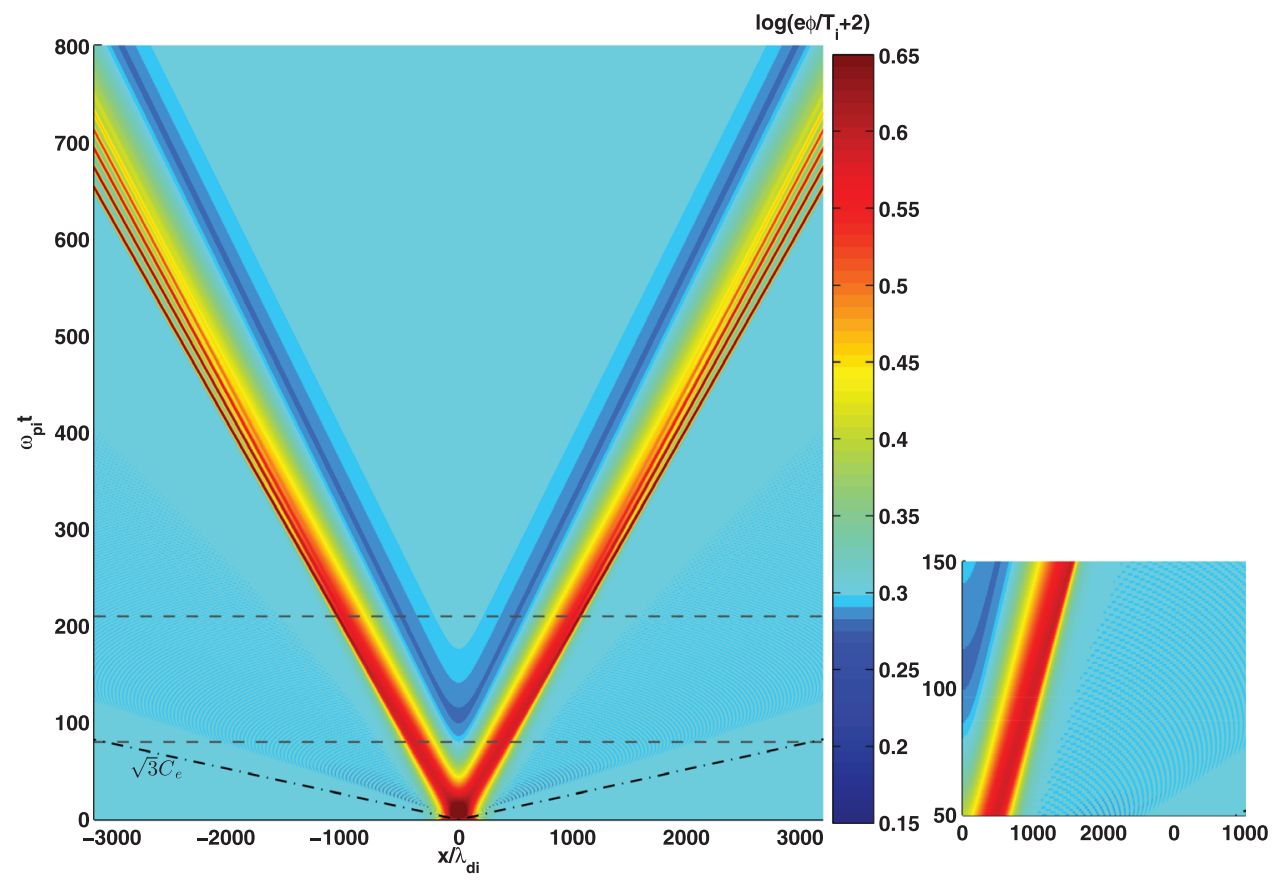

FIG. 7. Spatial and temporal evolution of electrostatic potentials for $l_{0}=100$, $\Delta n=1$ (Run-7). The upper dark blue bands represent the propagation path of IA oscillations. The red bands correspond to chain of IA pulses propagating opposite to each other. The inset shows Langmuir wave packet with distinct waves. The horizontal dotted lines at $\omega_{p i} t=90$ and $\omega_{p i} t=210$ correspond to formation of two unstable IA pulses and initiation of wave breaking, respectively.

(a)

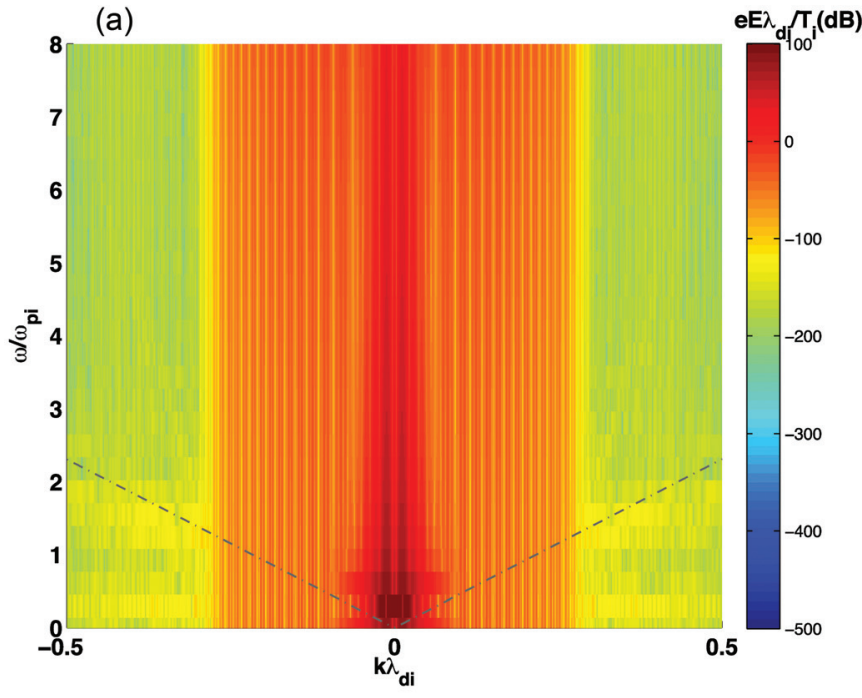

(c)

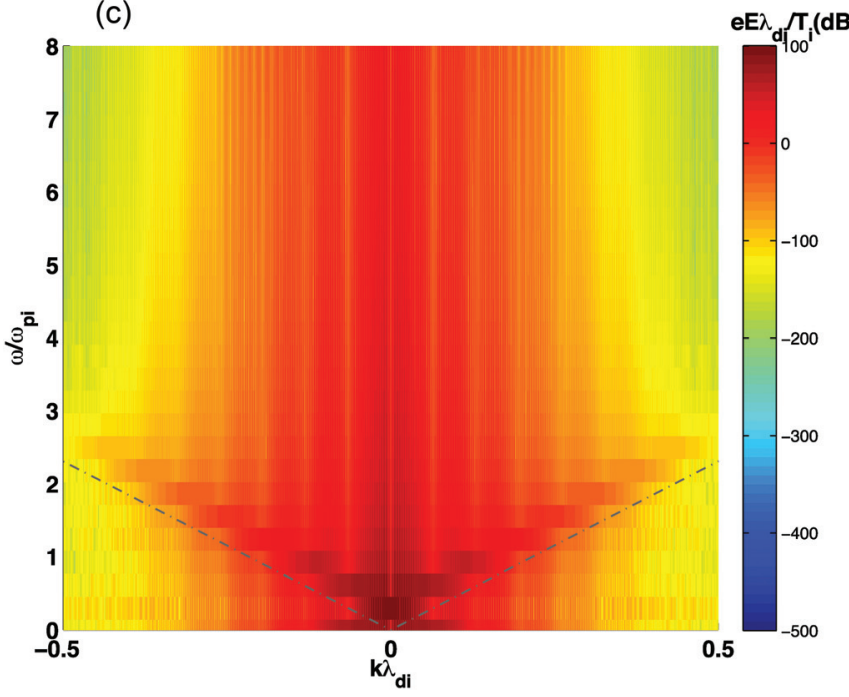

(b)

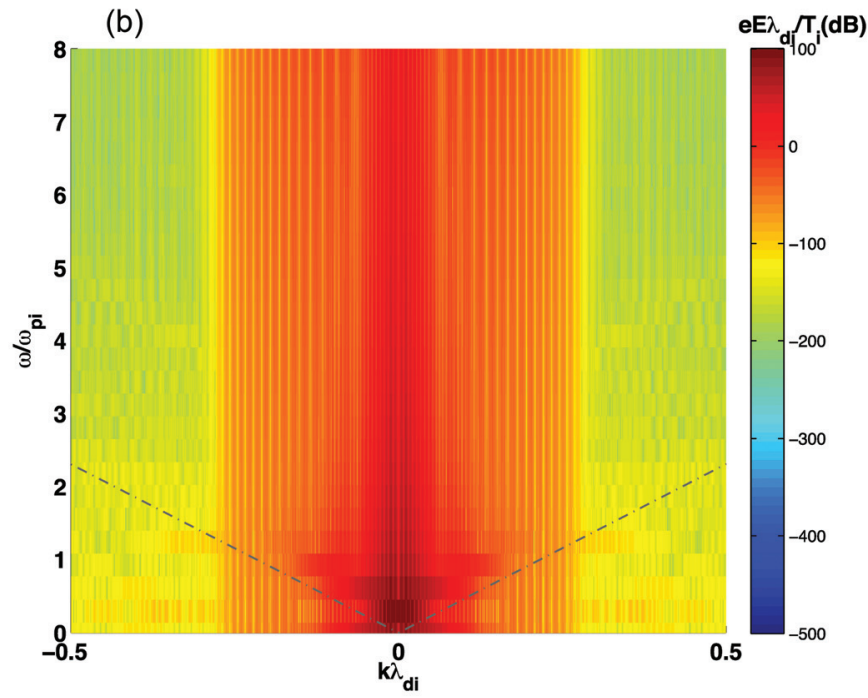

(d)

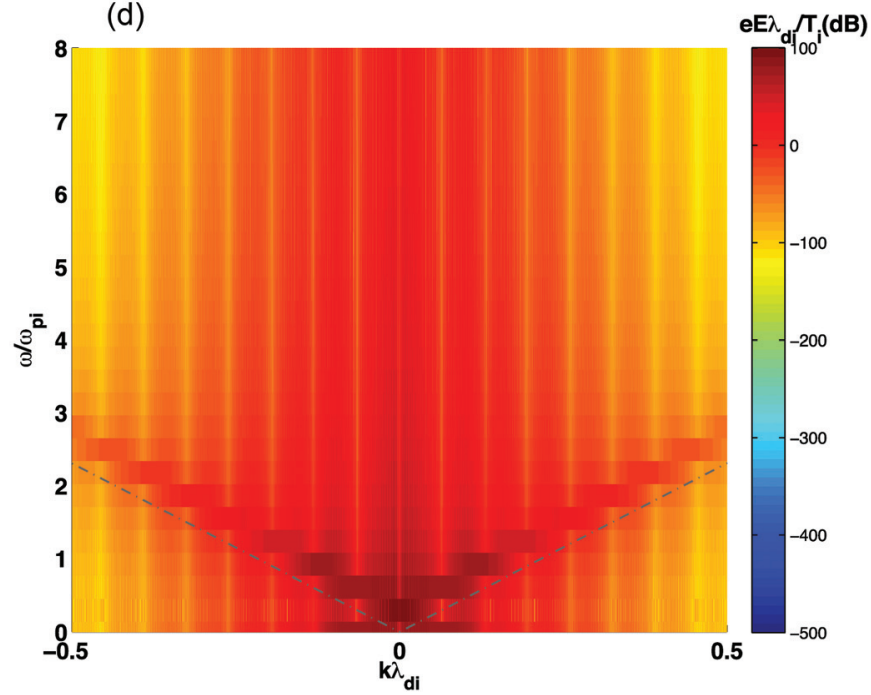

FIG. 8. $\omega-k$ dispersion diagrams during (a) $\omega_{p i} t=20-40$, (b) $\omega_{p i} t=80-100$, (c) $\omega_{p i} t=160-180$, and (d) $\omega_{p i} t=240-260$ for the simulation Run-7. The dispersion curves show IA mode. The slanted dotted lines represent the average speed $\left\langle V_{s}\right\rangle$ of fastest IA soliton in the chain during their stability. 
dispersion diagrams during wave steepening and after wave breaking, respectively. In these figures, the two dispersion curves are linked with the IA mode. Although Langmuir dispersion curves are present at $\omega / \omega_{p i}=10$, they are not visible as we are focusing on the evolution of ion acoustic dispersion. It is observed that the IA mode is associated with smaller values in $k$-space during the early phase of evolution. However, the mode is found to be associated with the larger values in $k$-space during the process of wave steepening and wave breaking process, which indicates the development of short wavelength structures in the system.

The speeds of solitons in the chain are greater than the those of IA oscillations, $V_{s 0}$. The solitary pulses formed in the chain become distinct due to their difference in propagation speeds and evolve into the chain of stable IA solitons at time $\omega_{p i} t=5800$. The speed, amplitude, and width of each IA soliton of the chain in their stability region are estimated and given in Table II. To demonstrate the stability of the IA solitons, we have shown snapshots of the soliton chain propagating in positive $x$-direction at $\omega_{p i} t=5800$ and $\omega_{p i} t=6800$ in Figure 9. Figures 9(a)-9(c), respectively, show the electron and ion densities, electric field, and electrostatic potential of the solitary chain at time $\omega_{p i} t=5800$. Likewise, the same parameters at $\omega_{p i} t=6800$ are shown in Figures 9(d)-9(f). The snapshots at two different times show that the amplitude of densities, electric field, and the electrostatic potential associated with each solitons in the solitary chain are nearly the same, which reveals the stability of all the solitons in the solitary chain.

\section{NONLINEAR FLUID THEORY OF IA SOLITONS}

The Sagdeev pseudopotential method ${ }^{30}$ is widely used to study arbitrary amplitude solitary waves analytically in a stationary reference frame. Here, we develop the nonlinear fluid theory of IA solitons for electron-ion plasma using the Sagdeev pseudopotential technique to compare it with fluid simulations.

We use Eqs. (1)-(4) and assume that all variables are functions of a single variable, $\xi=(x-M t)$. We transform these equations to a stationary frame moving with the phase velocity of the solitary wave, $V_{s}$. Here, $M=V_{s} / C_{i}$ is the Mach number, which is a measure of a normalized velocity of an IA soliton. Then, solving for perturbed densities, putting these expressions in the Poisson equation, and assuming appropriate boundary conditions for the localized disturbances along with the conditions that $\phi=0$ and $d \phi / d \xi=0$ at $\xi \rightarrow \pm \infty$, we obtain the following energy integral: ${ }^{34,35}$

$$
\frac{1}{2}\left(\frac{\partial \phi}{\partial \xi}\right)^{2}+\psi(\phi, M)=0
$$

where

$$
\begin{aligned}
\psi(\phi, M)= & \mu_{e} n_{e 0}\left\{M^{2}-\frac{M}{\sqrt{2}} B_{e}^{1 / 2}\right\}+n_{e 0} T_{r}\left\{1-2 \sqrt{2} M^{3} B_{e}^{-3 / 2}\right\} \\
& +n_{i 0}\left\{M^{2}-\frac{M}{\sqrt{2}} B_{i}^{1 / 2}\right\}+n_{i 0}\left\{1-2 \sqrt{2} M^{3} B_{i}^{-3 / 2}\right\}
\end{aligned}
$$

is the pseudopotential, also known as the Sagdeev potential. Here,

$$
\begin{aligned}
& B_{e}=A_{e} \pm \sqrt{A_{e}^{2}-\frac{12 T_{r} M^{2}}{\mu_{e}}}, \quad B_{i}=A_{i} \pm \sqrt{A_{i}^{2}-12 M^{2}}, \\
& A_{e}=M^{2}+\frac{3 T_{r}}{\mu_{e}}+\frac{2 \phi}{\mu_{e}}, \quad A_{i}=M^{2}+3-2 \phi, \\
& \mu_{e}=\frac{m_{e}}{m_{i}}, \quad T_{r}=\frac{T_{e}}{T_{i}} .
\end{aligned}
$$
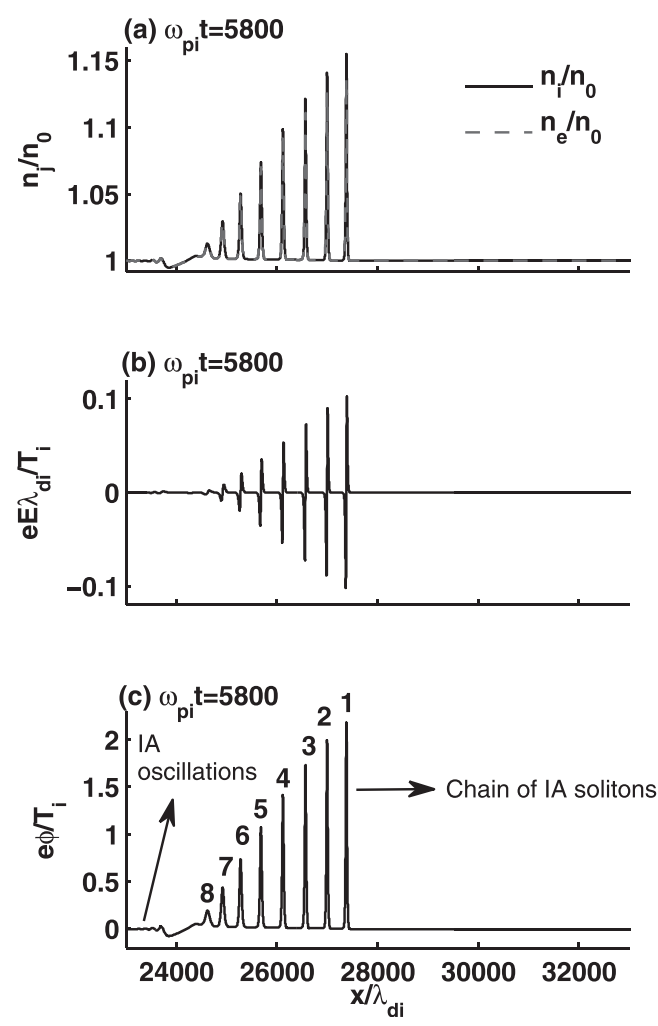

(d) $\omega_{p i} t=6800$

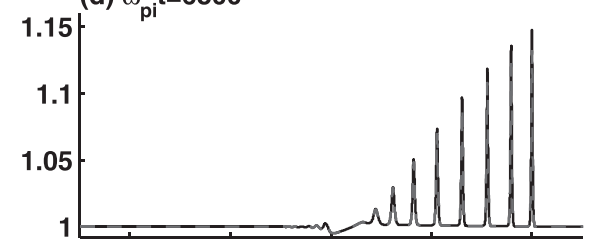

(e) $\omega_{p i} t=6800$

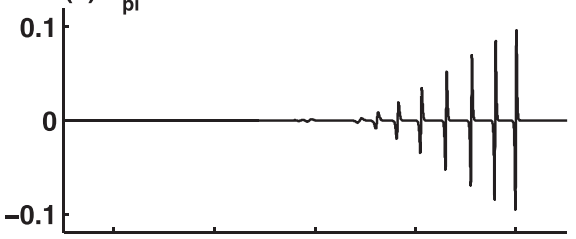

FIG. 9. Snapshots of (a) electron and ion densities (b) electric field and (c) electrostatic potential associated with chains of IA solitons for $\omega_{p i} t=5800$ for Run-7. The same parameters are shown in (d)-(f) for $\omega_{p i} t=6800$. Here, the profiles are associated with the solitons propagating in positive $x$-direction. 
Equation (10) describes the motion of a pseudo particle of a unit mass in a pseudo potential $\psi$, where $\phi$ and $\xi$ play the role of displacement $x$ from the equilibrium and time $t$, respectively. Equation (10) yields solitary wave solutions when the Sagdeev potential satisfies the following conditions:

$$
\begin{aligned}
& \psi(\phi, M)=0, d \psi(\phi, M) / d \phi=0, \\
& d^{2} \psi(\phi, M) / d \phi^{2}<0 \text { at } \phi=0, \\
& \psi(\phi, M)=0 \text { at } \phi=\phi_{\max }, \text { and } \\
& \psi(\phi, M)<0 \text { for } 0<|\phi|<\left|\phi_{\max }\right| .
\end{aligned}
$$

It is clear from Eq. (11) that $\psi(\phi, M)$ and its first-order derivative with respect to $\phi$ vanishes at $\phi=0$, thus Eq. (11) always satisfies the first two conditions mentioned above. The third condition, i.e., $d^{2} \psi(\phi, M) / d \phi^{2}<0$ at $\phi=0$, is fulfilled only if $M>M_{0}$, where $M_{0}$ satisfy the equation

$$
f\left(M_{0}\right) \equiv \frac{n_{e 0}}{\mu_{e}\left[M_{0}^{2}-\frac{3 T_{r}}{\mu_{e}}\right]}+\frac{n_{i 0}}{\left[M_{0}^{2}-3\right]}=0 .
$$

For each $T_{r}$, the numerical solution of Eq. (12) in general gives two critical Mach numbers $\pm M_{0}$, which indicate the presence of two IA solitons with equal and opposite speeds. $M_{0}$ is the critical mach number and soliton solutions exist only for $M>M_{0}$. For $\operatorname{Tr}=5, \mu_{e}=0.01$, and $n_{e 0}=n_{i 0}=1$, we get $M_{0}= \pm 4.22$. Thus, for particular $M>M_{0}$, soliton solutions can be obtained by solving Eq. (10) numerically.

\section{COMPARISON: FLUID SIMULATION VS. NONLINEAR FLUID THEORY}

To compare the simulation results with the nonlinear fluid theory, we summarize the properties of IA solitons in the stability region (i.e., $\omega_{p i} t=t_{1}$ to $t_{2}$ ) for different simulation runs. We estimate the averages of physical quantities, such as amplitude $\left(\left\langle\phi_{m}\right\rangle\right)$, width $(\langle w\rangle)$, and speed $\left(\left\langle V_{s}\right\rangle\right)$ of each soliton propagating in positive $x$-direction, and these details are given in Table II. The standard deviations of the average speed and amplitude of the solitons are$\sim 0.2 \%$ and $0.4 \%$, respectively. This implies that the formed solitons are considerably stable during their propagation from $\omega_{p i} t=t_{1}$ to $t_{2}$. It should be noted that for a short (long) wavelength IDP, two identical single solitons (chains of multiple solitons) are formed. The number of solitons $\left(N_{\text {sol }}\right)$ established in each simulation run, which are travelling in positive $x$-direction, is also provided in Table II along with the speed of IA oscillations following them. Therefore, for each run, the total number of solitons formed in the simulation system is $2 N_{\text {sol }}$.

The number of solitons formed in the solitary chains is found to increase with the strength of the initial density perturbation. For perturbation strengths $S=24,40,120$, and 200 , the numbers of solitons formed in each solitary chain are, respectively, 2, 3, 5, and 8. The large perturbation strength produces large amplitude solitons with greater speeds as compared to those IA solitons that are formed with small perturbation strengths. It is observed that the speed of IA oscillations, $V_{s 0}$ obtained from the simulation is less than or equal to the critical mach number, $M_{0}$ computed from the nonlinear fluid theory. Also the speeds of all IA solitons are found to be greater than the speed of IA oscillations, i.e., $V_{s 0} \leq V_{s}$. We use the average soliton speed $\left\langle V_{s}\right\rangle$ as an input to the nonlinear fluid model and solve Eq. (10) numerically to obtain a soliton profile. The amplitudes and widths of these soliton profiles are given in Table II.

The $\left\langle\phi_{m}\right\rangle$ and $\langle w\rangle$ estimated from the simulations are plotted as functions of $\left\langle V_{s}\right\rangle$ along with their corresponding values of $\phi_{m}$ and $w$ incurred from nonlinear fluid theory in Fig. 10. It is seen that the properties of stable solitons
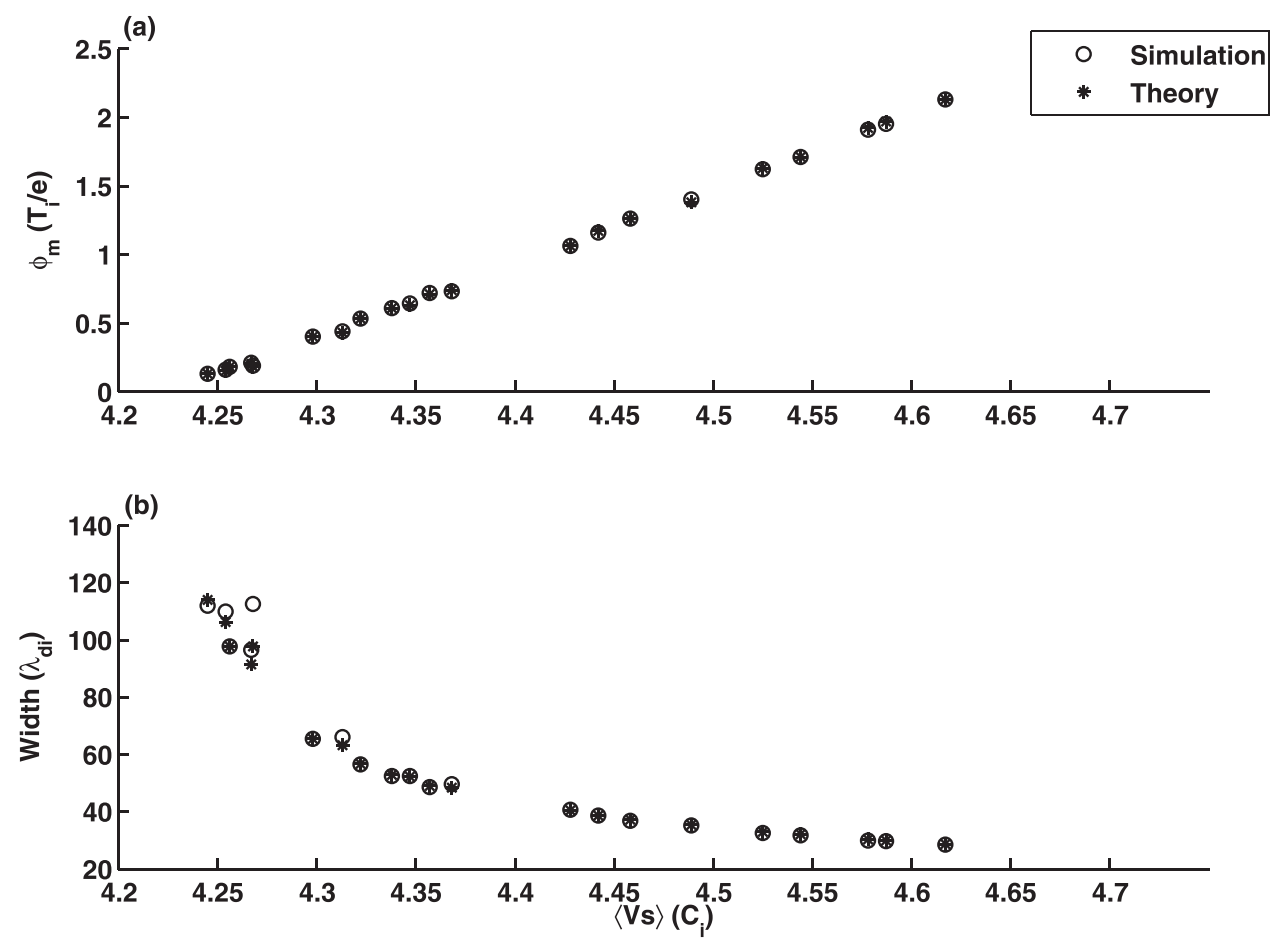

FIG. 10. Plots of average amplitude and width as a function of speed for IA solitons established in the simulation system during their stability along with the amplitude and width obtained from nonlinear fluid theory for respective speeds. 
established in the fluid simulations for both long and short wavelength IDPs are in good agreement with those provided by the nonlinear fluid theory. Thus, our fluid simulations validate the nonlinear fluid theory and confirm the stability of soliton solutions provided by this theory. It is noted that for $\left\langle V_{s}\right\rangle=4.27$, the maximum amplitude and width obtained from the theory deviate considerably from those estimated from the simulations. This solitary pulse is the smallest amplitude pulse (soliton number 8 ) in the solitary chain of Run7. During $\omega_{p i} t=5800-6800$, it is seen that this pulse is still evolving and needs more time to acquire stability. However, we do not have simulation data after $\omega_{p i} t=6800$ to check its stability.

\section{WAVE BREAKING OF UNSTABLE IA SOLITARY PULSES}

Generally, in fluid dynamics, it is observed that a breaking wave approaches a critical amplitude before the initiation of wave breaking. When the wave amplitude tends to approach the critical amplitude (or wave breaking amplitude), the wave steepens, and deformation occurs at the wave crest either at the leading or trailing edge. This wave steepening results in formation of short wavelength pulses. The similar mechanism is observed in our fluid simulations during the generation of IA solitons in case of a long wavelength type IDP.

We compute the electrostatic energy $U_{E}=E^{2} / 2 L_{x}$ and kinetic energy of the electron $K E_{e}=n_{e} m_{e} v_{e}^{2} / 2 L_{x}$ per unit system length for all simulation runs. Figures 11(a)-11(d) show $U_{E}$ and $K E_{e} / 2$ as functions of time for the simulation runs with long wavelength IDP Run-4, Run-5, Run-6, and Run-7, respectively. At the early phase of evolution, the electrostatic energy increases and it is greater than half of the kinetic energy of the electrons. At the later time, this electrostatic energy decreases and remains lower than $K E_{e} / 2$. However, the electrostatic energy again enhances significantly and exceeds $K E_{e} / 2$. This scenario is observed only for long wavelength type density perturbations, which is linked with the wave breaking process. The electrostatic potential profile of IA solitary pulses traveling in positive $x$-direction corresponding to the time close to $U_{E}=K E_{e} / 2$ are shown in Figures 11(e)-11(h) for respective simulation runs. It is noticed that the wave breaking process initiates close to the time when $U_{E}$ exceeds $K E_{e} / 2$. Also the amplitudes of collapsed solitary structures are found to be larger for $S=200$ (Run-7) and smaller for $S=24$ (Run-4). This implies that the wave breaking amplitude is determined by the strength of IDP. For $l_{0}=100, \Delta n=1$ (Run-7) and $l_{0}=60, \Delta n=1$ (Run-6), the $U_{E}$ exceeds $K E_{e} / 2$ close to $\omega_{p i} t=210$ and $\omega_{p i} t=120$, respectively. Similarly, for $l_{0}=100, \Delta n=0.2$ (Run-5) and $l_{0}=60, \Delta n=0.2$ (Run-4), the $U_{E}$ exceeds $K E_{e} / 2$ close to $\omega_{p i} t=990$ and $\omega_{p i} t=420$, respectively. It indicates that the wave breaking occurs at a later time for a greater $l_{0}$ while $\Delta n$ is kept fixed, whereas it takes place at an earlier time for larger $\Delta n$ when $l_{0}$ is kept fixed. Thus, we conclude here that the wave breaking amplitude and the time of initiation of the wave breaking are both dependent on parameters, $l_{0}$ and $\Delta n$ of the long wavelength type IDP.

Figures 12(a)-12(c), respectively, show plots of $U_{E}$ and $K E_{e} / 2$ for Run-1, Run-2, and Run-3 which falls under the short wavelength type density perturbation. We have not observed the wave breaking process during the formation of IA solitons for these cases. Moreover, the electrostatic energy always remains greater than $K E_{e} / 2$. The time evolutions of the electrostatic energy and the kinetic energy of
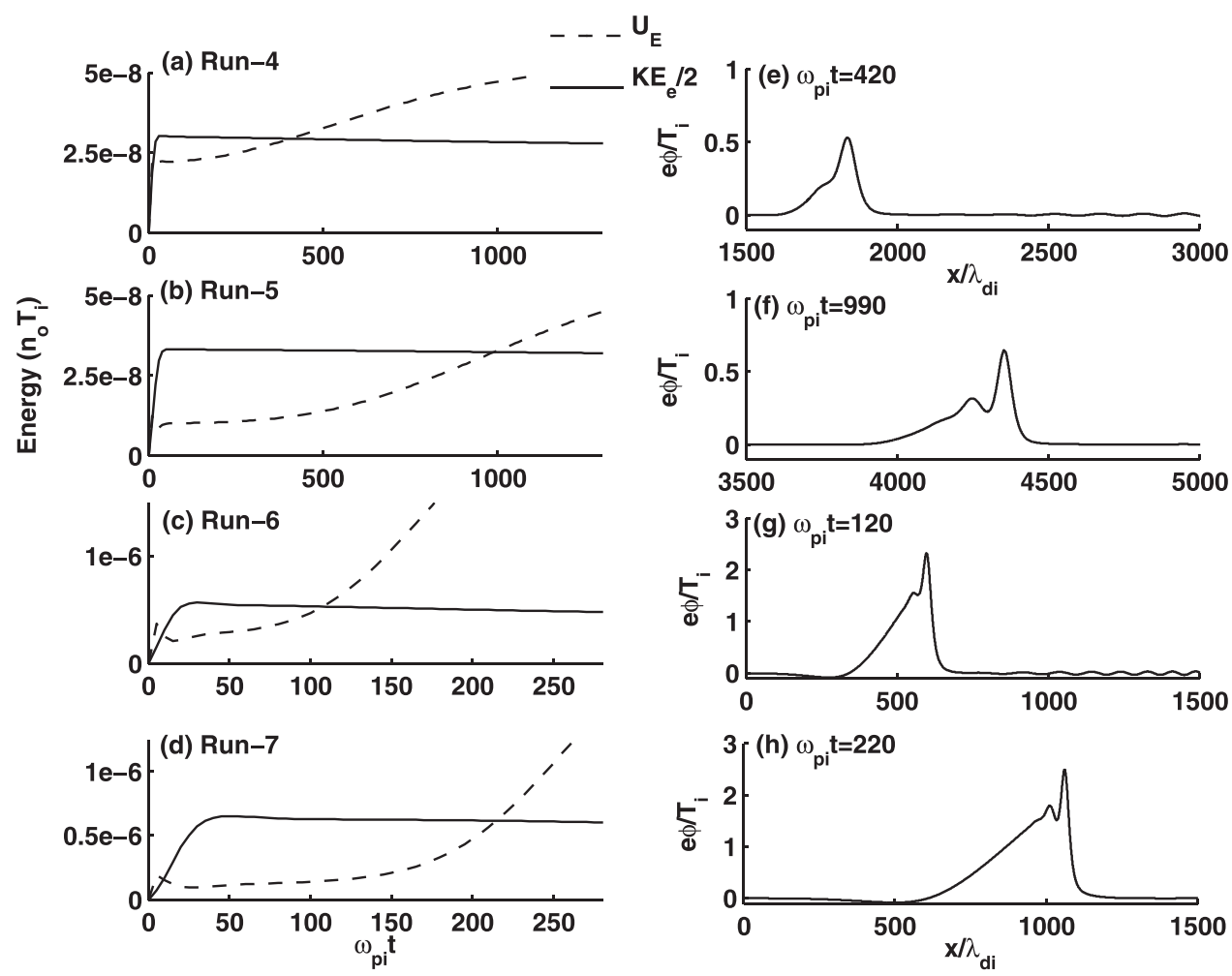

FIG. 11. Time evolution of electrostatic energy $U_{E}$ and half of kinetic energy of electrons $K E_{e} / 2$ for the long wavelength IDP simulations (a) Run-4, (b) Run-5, (c) Run-6, and (d) Run-7. The profile of electrostatic potential (propagating in positive $x$-direction) at time close to $U_{E}=K E_{e} / 2$ for respective simulation runs (e) Run-4, $\omega_{p i} t=420$, (f) Run-5, $\omega_{p i} t=990(\mathrm{~g})$ Run-6, $\omega_{p i} t=120$, and (h) Run-7, $\omega_{p i} t=220$. 

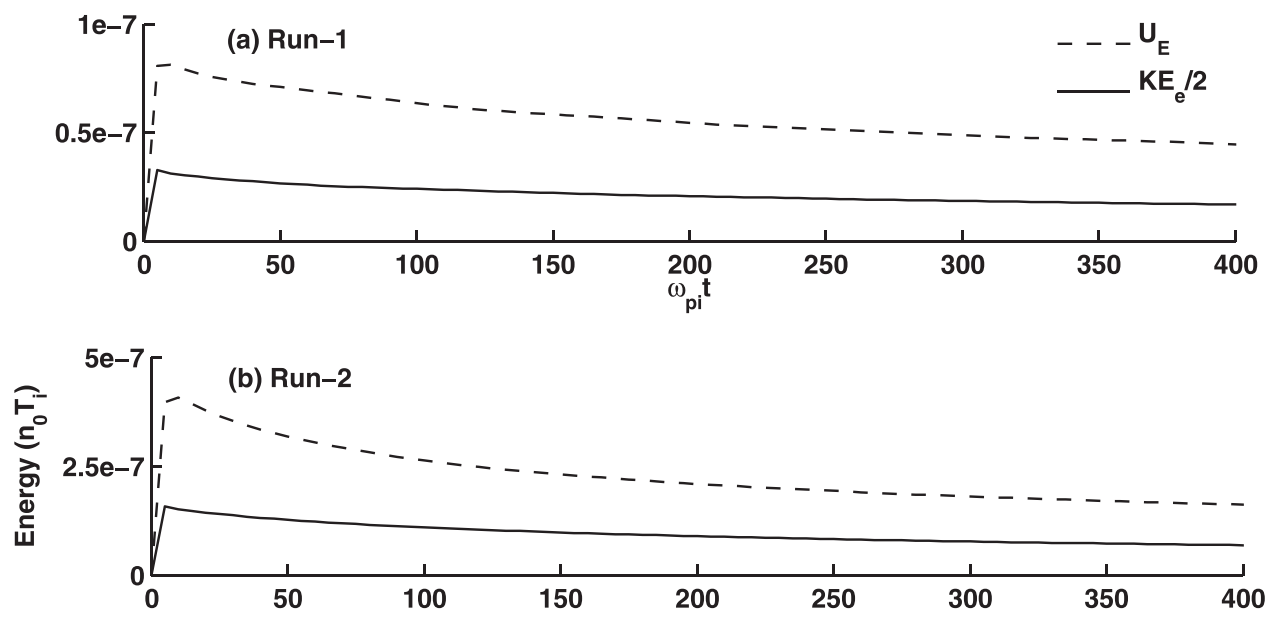

FIG. 12. Time evolution of electrostatic energy $U_{E}$ and half of kinetic energy of electrons $K E_{e} / 2$ for short wavelength IDP simulations (a) Run-1, (b) Run-2, and (c) Run-3.

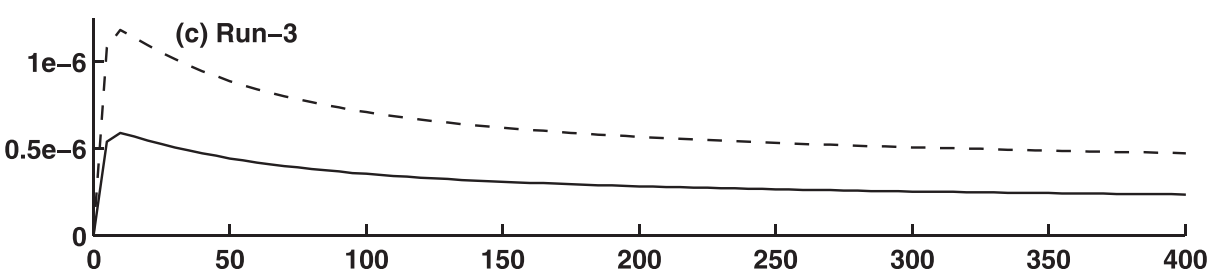

electrons are different as compared to the cases of long wavelength type density perturbation. This confirms that the condition of $U_{E}>K E_{e} / 2$ is observed during evolution of IA solitary pulses generated only with the long wavelength type density perturbation.

Generally, any perturbed system evolves with time and try to attain stability. In our fluid simulations for both long wavelength and short wavelength IDPs, stable IA solitons are formed in the system after a long-time nonlinear evolution. The solitons are stable solitary structures that propagate with constant speeds when the dispersive effects balance the nonlinearity in the medium. The linear dispersion relation for IA mode is

$$
\frac{\omega}{k}=\sqrt{\frac{\gamma_{e} T_{e}}{m_{i}\left(1+k^{2} \lambda_{d e}^{2}\right)}+\frac{\gamma_{i} T_{i}}{m_{i}}} .
$$

The equation above suggests that the phase speed of IA mode is dependent on wave numbers for $k^{2} \lambda_{d e}^{2} \gg 1$. In such cases, the stable soliton solutions are possible as the dispersive effects are present in the medium to balance the nonlinear effects. In view of this, the short wavelength IDP has a strong dispersive effect, whereas long wavelength IDP has a weak dispersive effect. In the latter case, the initially formed IA solitary pulses break into the short wavelength structures. This wave breaking process introduces sufficient dispersive effects in the medium to balance the nonlinearity and the chains of stable IA solitons are formed.

\section{DISCUSSION AND CONCLUSIONS}

We have performed one-dimensional fluid simulations of the IA solitary waves propagating parallel to the magnetic field in an electron-ion plasma by considering a large system length. We find that the IDP in the plasma evolves into IA solitons, IA oscillations, and Langmuir waves. This evolution process is found to be depend on the wavelength of the IDP. The short wavelength $k^{2} \lambda_{d e}^{2}>1$ type IDP evolves into two identical oppositely propagating single solitons, whereas the long wavelength $k^{2} \lambda_{d e}^{2}<1$ type IDP evolves into two identical oppositely propagating chains of multiple solitons. A similar phenomenon of formation of solitons is observed experimentally in the University of California at Los Angeles double plasma device, ${ }^{44}$ in the fluid simulation, ${ }^{45}$ and also in a numerical simulation of the $\mathrm{KdV}$ equation using a finite difference approach. ${ }^{28}$

It is seen that the wave breaking process plays an important role in the formation of chains of multiple IA solitons and it is found to take place only with the long wavelength $\left(k^{2} \lambda_{d e}^{2}<1\right)$ type IDPs. Also it is observed that the number of solitons formed in the solitary chain increases with the increasing strength $S$ of the IDP. These IA solitons are similar to those obtained from the $\mathrm{KdV}$ equation with respect to their shape and velocity of the soliton. ${ }^{28}$

It is known that propagation of the nonlinear plasma waves has a fundamental limitation given by the wave breaking threshold. In general, the solitary pulse generated in a fluid tries to acquire the maximum amplitude before they start to collapse through the wave breaking process. In the past studies, it is reported that the longitudinal wave breaking in a cold one-dimensional plasma occurs when elements of the plasma electron fluid that started out in different positions overtake each other while moving back and forth during the passage of the wave. ${ }^{46}$ This overtaking happens when the peak fluid velocity equals the phase speed of the plasma wave. In a warm plasma, the thermal effects modify the wave breaking amplitude. ${ }^{47,48}$ In this context, the results presented here indicate that the wave breaking amplitude is determined by the strength of the IDP. The greater the perturbation strength is, the larger the wave breaking amplitude of the IA solitary wave is in the warm plasma. Also, the time at which the wave breaking process initiates is found to be 
dependent on the perturbation parameters $\Delta n$ and $l_{0}$. For a fixed perturbation width $\left(l_{0}\right)$, the wave breaking occurs earlier for larger density perturbations; while for the fixed density perturbations, it occurs at earlier time for smaller widths. This indicates that the time of occurrence of the wave breaking depends on characteristics of the IDP. It is noticed that electrostatic energy and kinetic energy of the electron fluid satisfy the condition $\left(U_{E}>K E_{e} / 2\right)$ close to the time of initiation of the wave breaking.

The nonlinear fluid theory for IA solitons is developed by employing the Sagdeev pseudo potential approach and the results are compared with the simulations. This theory predicts two identical IA solitons with Mach numbers $\pm M$, which implies that these solitons propagate in opposite directions with the same speed $(|M|)$, which is consistent with the present fluid simulations.

The speeds of IA solitons obtained from the fluid simulations are found to be greater than the critical Mach $\left(M_{0}\right)$ number estimated from the nonlinear fluid theory. This is in accordance with the nonlinear fluid theory that supports the existence of IA soliton solutions for Mach numbers greater than $M_{0}$. The average amplitude and width of IA solitons computed from the simulations are in good agreement with those obtained from the nonlinear fluid theory.

The fluid simulations presented in this paper are the first ensuring the result from the general fluid theory, which has been extensively applied in interpretations of the solitary wave observations in terms of electron and IA solitary waves in space and laboratory plasmas.

It is noted that the Sagdeev pseudo potential method predicts only the stationary IA solitary solutions, where the soliton conditions are accomplished. It does not provide information about the transition process through which the stable IA solitons are generated in the electron-ion plasma. The present simulation uses the standard initial perturbation in equilibrium electron and ion densities, which evolves into IA solitons in the plasma. Such localized perturbations are produced in a laboratory plasma by very high laser powers and are known to be responsible for the generation of solitary waves in plasmas. ${ }^{49}$ In space, such density perturbations can originate in the Earth's magnetospheric regions, such as bow shock, magnetopause, and magnetosheath driven by solar wind variations. ${ }^{50}$ Extension of the present one-dimensional simulation to two- or three-dimensional models in magnetized plasmas is left as future studies.

\section{ACKNOWLEDGMENTS}

The computation was performed with supercomputers at Research Institute for Sustainable Humanosphere and Academic Center for Computing and Media Studies of Kyoto University. A.K. is supported by Grant-in-Aid 23.01024 for JSPS fellows.

\footnotetext{
${ }^{1}$ A. R. Osborne and T. L. Burch, Science 208, 451 (1980).

${ }^{2}$ A. Modena, Z. Najmudin, A. E. Dangor, C. E. Clayton, K. A. Marsh, C. Joshi, V. Malka, C. B. Darrow, C. Danson, D. Neely, and F. N. Walsh, Nature (London) 377, 606 (1995).
}

${ }^{3}$ K. E. Strecker, G. B. Partridge, A. G. Truscott, and R. G. Hulet, Nature 417, 150 (2002).

${ }^{4}$ G. I. Stegeman and M. Segev, Science 286, 1518 (1999).

${ }^{5}$ H. Matsumoto, H. Kojima, T. Miyatake, Y. Omura, M. Okada, I. Nagano, and M. Tsutui, Geophys. Res. Lett. 21, 2915, doi:10.1029/94GL01284 (1994).

${ }^{6}$ R. E. Ergun, C. W. Calrson, J. P. McFadden, F. S. Mozer, G. T. Delory, W. Peria, C. C. Chaston, M. Ternerin, I. Roth, L. Muschietti, R. Elphic, R. Strangeway, R. Pfaff, C. A. Cattell, D. Klumpar, E. Shelley, W. Peterson, E. Moebius, and L. Kistler, Geophys. Res. Lett. 25, 2041, doi:10.1029/ 98GL00636 (1998).

${ }^{7}$ J. S. Pickett, S. W. Kahler, L. J. Chen, R. L. Huff, O. Santolik, Y. Khotyaintsev, P. M. E. Décréau, D. Winningham, R. Frahm, M. L. Goldstein, G. S. Lakhina, B. T. Tsurutani, B. Lavraud, D. A. Gurnett, M. André, A. Fazakerley, A. Balogh, and H. Réme, Nonlinear Process. Geophys. 11, 183 (2004).

${ }^{8}$ G. O. Ludwig, J. L. Ferreira, and Y. Nakamura, Nonlinear Process. Geophys. 52, 275 (1984).

${ }^{9}$ J. D. Moody and C. F. Driscoll, Phys. Plasmas 2, 4482 (1995).

${ }^{10}$ B. Lefebvre, L. J. Chen, W. Gekelman, P. Kintner, J. Pickett, P. Pribyl, and S. Vincena, Nonlinear Process. Geophys. 18, 41 (2011).

${ }^{11}$ V. A. Turikov, Phys. Scr. 30, 73 (1984).

${ }^{12}$ H. Schamel, Phys. Rep. 140, 161 (1986).

${ }^{13}$ A. Luque and H. Schamel, Phys. Rep. 415, 261 (2005).

${ }^{14}$ W. Lotko and C. F. Kennel, J. Geophys. Res. 88, 381, doi:10.1029/ JA088iA01p00381 (1983).

${ }^{15}$ N. Dubouloz, R. Pottelette, M. Malingre, and R. A. Treumann, Geophys. Res. Lett. 18, 155, doi:10.1029/90GL02677 (1991).

${ }^{16}$ M. Berthomier, R. Pottelette, and M. Malingre, J. Geophys. Res. 103, 4261, doi:10.1029/97JA00338 (1998).

${ }^{17}$ R. Pottelette, R. E. Ergun, R. A. Treumann, M. Berthomier, C. W. Carlson, J. P. McFadden, and I. Roth, Geophys. Res. Lett. 26, 2629, doi:10.1029/1999GL900462 (1999).

${ }^{18}$ I. B. Bernstein, J. M. Greene, and M. D. Kruskal, Phys. Rev. 108, 546 (1957).

${ }^{19}$ Y. Omura, H. Kojima, and H. Matsumoto, Geophys. Res. Lett. 21, 2923, doi:10.1029/94GL01605 (1994).

${ }^{20}$ Y. Omura, H. Matsumoto, T. Miyake, and H. Kojima, J. Geophys. Res. 101, 2685, doi:10.1029/95JA03145 (1996).

${ }^{21}$ M. V. Goldman, M. M. Oppenheim, and D. L. Newman, Geophys. Res. Lett. 26, 1821, doi:10.1029/1999GL900435 (1999).

${ }^{22}$ N. Singh and G. Khazanov, J. Geophys. Res. 108, 8007, doi:10.1029/ 2002JA009436 (2003).

${ }^{23}$ D. A. Tidman and N. A. Krall, Shock Waves in Collisionless Plasmas (Wiley-Interscience, New-York, 1971), Chap. 6.

${ }^{24}$ N. Singh, J. Pure Appl. Phys. 11, 533 (1973).

${ }^{25}$ H. Washimi and T. Taniuti, Phys. Rev. Lett. 17, 996 (1966).

${ }^{26}$ N. Dubouloz, R. A. Treumann, R. Pottelette, and M. Malingre, J. Geophys. Res. 98, 17415, doi:10.1029/93JA01611 (1993).

${ }^{27}$ A. P. Kakad, S. V. Singh, R. V. Reddy, G. S. Lakhina, and S. G. Tagare, Adv. Space Res. 43, 1945 (2009).

${ }^{28}$ N. J. Zabusky and M. D. Kruskal, Phys. Rev. Lett. 15, 240 (1965).

${ }^{29}$ C. S. Gardner, J. M. Greene, M. D. Kruskal, and R. M. Miura, Phys. Rev. Lett. 19, 1095 (1967).

${ }^{30}$ R. Z. Sagdeev, Rev. Plasma Phys. 4, 23 (1966).

${ }^{31}$ S. V. Singh, R. V. Reddy, and G. S. Lakhina, Adv. Space Res. 28, 1643 (2001).

${ }^{32}$ A. P. Kakad, S. V. Singh, R. V. Reddy, G. S. Lakhina, S. G. Tagare, and F. Verheest, Phys. Plasmas 14, 052305 (2007).

${ }^{33}$ S. S. Ghosh, J. S. Pickett, G. S. Lakhina, J. D. Winningham, B. Lavraud, and P. M. E. Décréau, J. Geophys. Res. 113, A06218, doi:10.1029/ 2007JA012768 (2008).

${ }^{34}$ G. S. Lakhina, A. P. Kakad, S. V. Singh, and F. Verheest, Phys. Plasmas 15, 062903 (2008).

${ }^{35}$ G. S. Lakhina, S. V. Singh, A. P. Kakad, F. Verheest, and R. Bharuthram, Nonlinear Process. Geophys. 15, 903 (2008).

${ }^{36}$ G. S. Lakhina, S. V. Singh, A. P. Kakad, M. L. Goldstein, A. F. Vińas, and J. S. Pickett, J. Geophys. Res. 114, A09212, doi:10.1029/2009JA014306 (2009).

${ }^{37}$ T. K. Baluku, M. A. Hellberg, and F. Verheest, Europhys. Lett. 91, 15001 (2010).

${ }^{38}$ G. S. Lakhina, S. V. Singh, and A. P. Kakad, Adv. Space Res. 47, 1558 (2011).

${ }^{39}$ G. S. Lakhina, S. V. Singh, A. P. Kakad, and J. S. Pickett, J. Geophys. Res. 116, A10218, doi:10.1029/2011JA016700 (2011). 
${ }^{40}$ F. Verheest, M. A. Hellberg, and I. Kourakis, Phys. Plasmas 20, 012302 (2013).

${ }^{41} \mathrm{~K}$. Watanabe and T. Sato, High-Precision MHD Simulation, in Computer Space Plasma Physics: Simulation Techniques and Software (Terra Scientific, Tokyo Japan, 1993), Chap. 7, pp. 209-215.

${ }^{42}$ Y. Omura and J. L. Green, J. Geophys. Res. 98, 9189, doi:10.1029/ 92JA02901 (1993).

${ }^{43} \mathrm{~F}$. Chen, Introduction to Plasma Physics and Controlled Fusion, 2nd ed. (Springer, New-York, 1993), pp. 95-99.
${ }^{44}$ H. Ikezi, R. J. Taylor, and D. R. Baker, Phys. Rev. Lett. 25, 11 (1970).

${ }^{45}$ S. Baboolal, Math. Comput. Simul. 55, 309 (2001).

${ }^{46}$ J. M. Dawson, Phys. Rev. 113, 383 (1959).

${ }^{47}$ T. P. Coffey, Phys. Fluids 14, 1402 (1971).

${ }^{48}$ T. Katsouleas and W. B. Mori, Phys. Rev. Lett. 61, 90 (1988).

${ }^{49}$ T. E. Sheridan, V. Nosenko, and J. Goree, Phys. Plasmas 15, 073703 (2008).

${ }^{50}$ D. G. Sibeck and J. T. Gosling, J. Geophys. Res. 101, 31, doi:10.1029/ 95JA03141 (1996). 